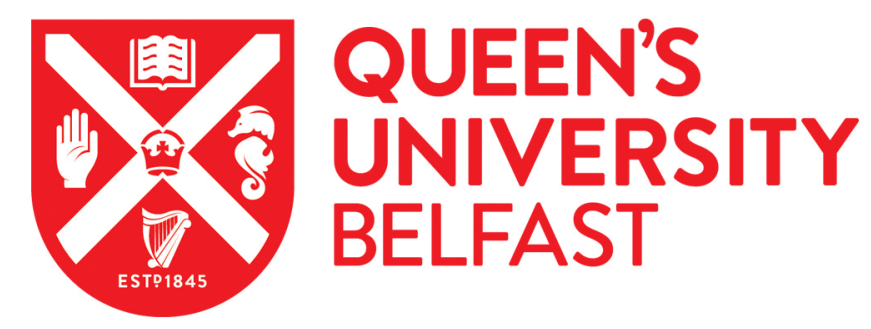

\title{
The Radiobiology of Proton Therapy Challenges and Opportunities Around Relative Biological Effectiveness
}

Jones, B., McMahon, S. J., \& Prise, K. M. (2018). The Radiobiology of Proton Therapy Challenges and Opportunities Around Relative Biological Effectiveness. Clinical oncology (Royal College of Radiologists (Great Britain)). https://doi.org/10.1016/j.clon.2018.01.010

Published in:

Clinical oncology (Royal College of Radiologists (Great Britain))

Document Version:

Peer reviewed version

Queen's University Belfast - Research Portal:

Link to publication record in Queen's University Belfast Research Portal

Publisher rights

Copyright 2018 Elsevier.

This manuscript is distributed under a Creative Commons Attribution-NonCommercial-NoDerivs License

(https://creativecommons.org/licenses/by-nc-nd/4.0/), which permits distribution and reproduction for non-commercial purposes, provided the author and source are cited

\section{General rights}

Copyright for the publications made accessible via the Queen's University Belfast Research Portal is retained by the author(s) and / or other copyright owners and it is a condition of accessing these publications that users recognise and abide by the legal requirements associated with these rights.

Take down policy

The Research Portal is Queen's institutional repository that provides access to Queen's research output. Every effort has been made to ensure that content in the Research Portal does not infringe any person's rights, or applicable UK laws. If you discover content in the Research Portal that you believe breaches copyright or violates any law, please contact openaccess@qub.ac.uk. 
Bleddyn Jones ${ }^{1}$, Stephen J. McMahon ${ }^{2}$ and Kevin M. Prise ${ }^{2}$

${ }^{1}$ Oxford Institute for Radiation Oncology, University of Oxford, Old Road Campus Research Building, Roosevelt Drive, Oxford, OX3 7DG, UK

${ }^{2}$ Centre for Cancer Research \& Cell Biology, Queen's University Belfast, 97 Lisburn Road, Belfast, BT9 7AE, UK 


\begin{abstract}
With the current UK expansion of proton therapy, there is a great opportunity for clinical oncologists to develop a translational interest in the associated scientific base and clinical results. In particular, the underpinning controversy regarding the conversion of photon dose to proton dose by the relative biological effectiveness (RBE) must be understood, including its important implications. At the present time, the proton prescribed dose includes an RBE of 1.1 regardless of tissue, tumour and dose fractionation. A body of data has emerged against this pragmatic approach, including a critique of the existing evidence base, due to choice of dose, use of only acute-reacting in vivo assays, analysis methods, and the reference radiations used to determine the RBE.
\end{abstract}

Modelling systems, based on the best available scientific evidence, and which include the clinically useful BED concept have also been developed to estimate proton RBEs for different dose and linear energy transfer (LET) values. The latter reflect ionisation density which progressively increases along each proton track. Late reacting tissues such as the brain, where $\alpha / \beta=2$ Gy, show a higher RBE than 1.1 at low dose per fraction (1.2-1.8 Gy) at LET values used to cover conventional target volumes and can be much higher. RBE changes with tissue depth appear to vary depending on the method of beam delivery used.

To reduce unexpected toxicity, which does occasionally follow proton therapy, a more rational approach to RBE allocation, using a variable RBE which depends on dose per fraction and the tissue and tumour radiobiological characteristics such as $\alpha / \beta$ is proposed. 


\section{Introduction}

Radiotherapy is the major treatment modality for around $50 \%$ of all cancer treatments internationally and is continuing to go through a rapid phase of technological development. Intensity modulated radiotherapy, arc therapies, stereotactic therapy, image guidance and recently MRI-linacs all aim to deliver highly conformal beams to maximise tumour dose. For high energy photon exposures the high reproducibility and accuracy is driving the rapid testing of stereotactic and hypofractionated treatments combined with novel molecular and immunotargeting strategies [1]. For many years in parallel, proton therapy has been developing based on the physical properties of the Bragg curve. As of the end of 2015, 131,240 patients had been treated (Proton Therapy Cooperative Group (PTCOG)) across a wide range of tumour types and as of June 2017 there are 62 centres being built or planned internationally, including 2 NHS centres and 4 private centres in the UK (see www.ptcog.ch). Carbon ions are also currently used for therapy but only in a few centres internationally and at a much lower level than protons with by 2015, 19,376 patients having being treated (PTCOG).

All therapeutic interventions have risks and benefits. For new pharmacological agents, there is considerable legislation that demands satisfactory pre-clinical experimental studies before human exposures are allowed; the same is not true for radiation therapy, where the legal emphasis is on quality assurance relating to dose associated with particular techniques. However, radiotherapy evolved pragmatically with its scientific base of radiobiology providing phenomenological support, sometimes with useful applications through the use of relatively simple mathematical models. Clinical Oncologists will be familiar with how the biological effective dose (BED) and equivalent dose in 2 Gy (EQD-2) concepts have multiple uses and can be used to make radiotherapy much safer, for example dealing with gaps in treatment [2].

Most oncologists consider proton therapy to be an extension of conventional therapy and should be integrated within the present clinical service provisions. This viewpoint is highly desirable for involvement of 'site specialist' oncologists, but the knowledge base of practitioners must be increased to cover the salient physical and radiobiological aspects of proton therapy.

In the context of proton therapy, which is regarded as the most promising modern form of treatment in certain clinical situations, there is a significant body of existing radiobiology research, although it is limited in scope as far as the applicability to human exposures and paucity of information for late normal tissue reactions. In this short paper we will consider the challenges and opportunities around protons, focussing on Relative Biological Effectiveness.

Proton therapy offers delivery of radical treatment doses, often with reduced energy deposition in the body, and avoidance of unnecessary direct radiation doses to many organs due to the 
Bragg peak effect [1]. There are three potential disadvantages that must be overcome to optimise the safety and effectiveness of their use:

1. Bragg peak placement inaccuracy due to physical reasons such as heterogeneous tissue densities, patient movement, daily positioning, and beam delivery related factors such as lateral scattering of the beam.

2. Within Bragg peaks, energy is deposited as clustered rather than sparse ionisation events. This causes more complex DNA damage that is more difficult or impossible for enzymatic DNA repair mechanisms to restore, resulting in enhanced biological effects. These may be advantageous within a cancer, but possibly deleterious for normal tissues in the tumour vicinity and which require to be treated to full or nearfull dose to achieve local tumour control.

3. Currently, the medical prescription of proton therapy dose includes a $10 \%$ reduction in dose to all tumours and tissues to compensate for enhanced bioeffectiveness. This correction factor is being challenged, but to understand this issue, it is necessary to be familiar with the physics and biology terms associated with enhanced bio-effectiveness, and how this is modified by physical and biological characteristics.

\section{Underpinning physics and radiobiology}

Along individual radiation tracks, the closeness of ionisations is expressed by the Linear Energy Transfer (or LET), which essentially averages the energy released per micrometre distance and is reported in units of keV per micron. As protons enter the body at high energy and slow down, the ionization density and hence the LET increases delivering the characteristic Bragg peak shape.

The other essential definition is that of relative biological effectiveness (or RBE). It is formally defined as a ratio, and in the context of proton therapy is:

$R B E=\frac{\text { Dose of the reference megavoltage photon radiation }}{\text { Dose of proton therapy }}$, each dose achieving the same specified biological effect.

Protons are typically prescribed to patients at doses where the photon-equivalent RBEweighted dose (denoted as Gy (RBE)) matches the photon physical dose. As a result, the actual delivered physical proton dose is equal to the physical photon (or $\mathrm{X}$-ray) dose divided by the proton RBE, presently assumed to be 1.1 in all tissues and tumours, irrespective of dose per fraction. If this 1.1 value is incorrect, the prescribed doses will also be incorrect. 
LET and RBE are closely related, with RBE initially increasing approximately linearly with LET. At higher LETs this rate of increase slows and eventually reaches a turning point after which RBE falls, due to energy 'wasting'. The magnitude of the RBE is inversely related to the dose and is also related non-linearly to the intrinsic cellular radiosensitivities ( $\alpha$ and $\beta$ parameters explained below).

In proton therapy Bragg peaks are 'spread out' to cover the tumour: tissues receive a mixture of Bragg peak (high LET) and non-Bragg peak (low LET) regions, often resulting in average LET's around 1-2 keV. $\mu \mathrm{m}^{-1}$ in the tumour region, although can be higher and up to 8-10 $\mathrm{keV} . \mu \mathrm{m}^{-1}$, depending on field arrangements and technique used. Pencil beam delivery can result in higher average LETs [3], with perhaps additional bio-effects due to differences in how the protons are modulated compared with passively scattered (wider) beams.

The average LET of conventional megavoltage radiotherapy is around $0.2 \mathrm{keV} . \mu \mathrm{m}^{-1}$, which has an immediate implication that the mid SOBP LET may be around 6-9 times higher. Another complicating factor is that much of the research on LET and RBE (for protons and other forms of radiation) used low voltage $\mathrm{x}$-ray beams, whose LET was already around 1-1.5 keV. $\mu \mathrm{m}^{-1}$, and was frequently used to estimated proton RBEs despite introducing the risk of underestimating RBEs.

For further understanding it is necessary to describe the simple, but elegant mathematical relationship between radiation dose (d) and bio-effectiveness $(E)$ described by the linearquadratic (LQ) model (see Table 1):

Some general trends have been confirmed from LET-RBE experimental studies that have used a variety of radiation modalities, including fast neutrons (that mainly produce recoil protons), low energy x-rays, alpha particles, carbon, and other light ions [4-9]. These include the fact that the $\alpha$ parameter increases by more than the $\beta$ parameter with LET, which leads to the inverse relationship between RBE and dose. At very low dose per fraction the RBE will be close to the ratio $\alpha_{H} / \alpha_{L}$, (often referred to as the RBEmax), while at high doses the RBE falls, becoming closer and closer to the ratio $\sqrt{\beta_{H}} / \sqrt{\beta_{L}}$ (the RBEmin) [10]. These RBE limits are contained in the BED equation shown in table 1 and figure 1.

\section{Radiobiological data and modelling}

Significant radiobiological effort has determined the RBE responses of a range of in vitro cell culture modes which have been extensively reviewed by Paganetti [11]. These studies, although extensive, have significant limitations, specifically they have been performed on a limited range of 2D cell culture models and most have used high doses per fraction where RBE is lowest. There is also in vivo data, but again limited to a small range of predominantly 
acute early-reacting tissue assays such as the upper intestinal (ileal) crypt assay ( $\alpha / \beta=10 \mathrm{~Gy}$ ), acute skin and lung reactions (pneumonitis rather than fibrosis). What is clear from these studies is that the biological response varies significantly with LET and more specifically the underlying $\alpha / \beta$ values of the biological system being studied. The high $\alpha / \beta$ ratios of acutereacting systems (and for rapidly growing tumours) tend to have lower RBEs at low dose per fraction. Also, RBE by definition requires a reference radiation and one aspect of the variability from different datasets is that this has varied from different low LET radiations such as gammarays, $\mathrm{kV}$ and $\mathrm{MV} \mathrm{X}$-rays which in themselves have different effectiveness. It is clear from a clinical perspective that a high energy linac (4-6MV) is the most appropriate radiation to use for future RBE experiments. In the influential data set of Paganetti et al [12], many studies used low energy X-ray reference radiations, whose higher LETs led to reduced RBE observations. Within this data set, this was corrected for by scaling proton LETs to 'normalised' LETs, taking a $6 \mathrm{MV}$ linac as the baseline radiation quality, although the conversion factor used was not necessarily correct for late effects. In addition, many experiments were performed at high fractional doses (around 10-14 Gy, since these doses were used for treating uveal melanomas), which also highlighted the dependence of RBE on dose, with higher average doses leading to lower RBEs.

While the conclusion of this review was that an average proton RBE between 1.1 and 1.15 was a good approximation of the average across all results within the limited range of assays used, there was significant heterogeneity, which could lead to significant differences in clinical treatments [12]. Many other authors have expressed concern about the use of a constant RBE $[12,13-18]$. Experiments by various groups $[19,20]$ show unequivocal changes in RBE with dose per fraction at various positions along a proton beam (see figure 2). Radiobiology research has also highlighted that different variants of the same tumour type could potentially have different RBE values. For example Liu et al [21] have reported variable proton RBEs in a large panel of non-small cell lung tumour lines with deficiencies in the Fanconi's anemia/BRCA pathways predicting an increased RBE. There is also evidence that RBE can be influenced by the choice of DNA double-strand break repair pathway available [22] against a background of an understanding that many tumours may have compromised DNA repair pathways [23].

RBE changes with LET can be modelled using phenomenological or theoretical models or a combination of each [24-26]. They either relate RBE inversely to the $\alpha / \beta$ ratio of the control radiation, or involve separate treatment of $\alpha$, or $\alpha$ and $\beta$, coupled with saturation effects that limit the maximum possible increases. The later assumption, developed by Jones [10, 35], is used to generate figures 3 (a-c) for three $\alpha / \beta$ values (characteristic $\alpha / \beta$ values are: 2 Gy for the central nervous system, 5-12 Gy for many cancers and even higher for highly 
radiosensitive tumours such as lymphomas and childhood cancers). The baseline LET value is set to $0.22 \mathrm{keV} . \mu \mathrm{m}^{-1}$, being representative of clinical megavoltage photons.

It can be seen that the predicted RBE ranges could modify treatment outcomes considerably and it is reasonable to suggest that a greater dose reduction than $10 \%$ should be used within the CNS for volumes where LET values exceed $1.5 \mathrm{keV} . \mu \mathrm{m}^{-1}$.

Indeed figures 3 (a-c) not only show that RBE will change with dose per fraction, but also that the acute and late reacting systems with different $\alpha / \beta$ ratios effectively cross over. In other words, at low dose per fraction late-reacting tissues or tumours with low $\alpha / \beta$ ratios of 2-3 Gy have higher RBEs than acute-reacting tissues or tumours with high $\alpha / \beta$ ratios (7 Gy or more), but this order may be reversed at high dose per fraction, leading to lower RBEs in the late reacting tissues. Of the three examples in figure $3(\mathrm{a}-\mathrm{c})$, the first two represent LET values typically within spread-out Bragg peaks that cover a Planning Treatment Volume (PTV). The third (example $\mathrm{c}$ ) will be more typical of conditions further towards the end of range of proton fields, and without the PTV in some instances when using scanned beams [3]. Much higher LET (10-20 keV. $\mu \mathrm{m}^{-1}$ ) and consequently RBE values than given in (c) occur towards the end of the Bragg peaks $[10,35,37]$ with RBE's often greater than 2 . While there remain significant differences between the predictions of different models, clinical oncologists should compare these estimates with the standard value of 1.1 RBE at all doses per fraction and for all tissue types, and reflect on how these changes might influence normal tissue reactions, and it is important that the results of new radiotherapy techniques such as proton therapy are published with full assessment of late toxicities. Reports of higher than expected proton brain and brainstem toxicity, including radiological changes, have been reported [17, 27-29].

One centre has published a serious neurological toxicity (blindness and brain necrosis) of around $12.3 \%$ [30], a result that would be unacceptable after photon-based therapy. Also, long-term results of children treated with protons in Japan show significant grade 3 toxicities of $6 \%, 17 \%$ and $17 \%$ at five, ten and twenty years respectively [31].

Are these results due to Bragg peak placement errors or radiobiology uncertainties or perhaps both effects? Realistic assessment is needed. It is self-evident that errors in Bragg peak placement (away from the target) could result in reduced tumour control and be associated with increased normal tissue dose and so result in an increased risk of tissue side effects. Proton therapy dosimetry has significant additional challenges when compared to photon therapy, particularly at the end of range where even small shifts in patient setup or changes in anatomy can significantly impact on the dose delivered behind the target volume. As a result, there is significant research activity to deliver more accurate dose delivery information for protons [32]. However, range uncertainties arising from these effects are typically believed 
to be on the level of a few percent of the treatment dose/beam range in most cases. By contrast, experimental data and modelling has shown much greater uncertainties in proton RBE, up to nearly a factor of 2 for some tissues, suggesting that this may play a much more significant role in these 'unexpected' toxicities.

\section{Different radiobiology for different beam delivery systems?}

Proton beams are delivered using two alternate physical processes: (1) passive scattering where a metallic filter is used to create beam dispersion with inverse square fall off of intensity, which is then shaped using a second scatterer and collimator to deliver a uniform field at the target volume, (2) active scanning, where magnets are used to bend thin proton 'pencil beams' laterally and vertically to cover a target volume. Even at the same depth, the proton spectrum delivered can be quite different in the two systems. Since average LET is a linear measurement it does not reflect these differences, which can lead to differences in the density and complexity of tracks. Consequently, some index of LET distribution or track separation may also be required.

\section{Passively scattered beams}

There is evidence that if the same LET and dose is given at the same SOBP positions situated at markedly different depths, achieved by using a different incident energies, then the RBE is reduced in the deepest position [33]. These experiments used two different incident energies of 76 and $201 \mathrm{MeV}$, with mid SOBP's at $20.5 \mathrm{~mm}$ and $160 \mathrm{~mm}$ respectively, and SOBP distal ends at 26.9 and $185 \mathrm{~mm}$ respectively, each position with identical LET and dose characteristics. The only physical differences appeared to be the beam widths: $3 \mathrm{~cm}$ diameter at around $2 \mathrm{~cm}$ depth, and $12 \mathrm{~cm}$ diameter at $16 \mathrm{~cm}$ depth, showing greater lateral dispersion at the increased depth after passive beam scattering. In two human cell lines, the highest RBE's (1.3-1.33) at 2-3cm depth, reduced to be close to 1.0 at $16-18 \mathrm{~cm}$ depth. Although the dose rates reduced with increasing depth, the longer time cannot account for the RBE changes. A model of these results, using an exponential fit, is shown in figure 4.

\section{Actively scanned beams}

In contrast, [19] found no such effect on RBE with increasing depth in two cell lines with incident energies of 87 and $200 \mathrm{MeV}$ at Bloomington, but where scanned beams were used. Scanned beams, without metallic filter-related scatter, consist of essentially parallel tracks and so deliver potentially shorter inter-track separation distances $(s)$ over most of their range, with a narrower spread of particle energies. They exhibit little geometric dispersion until the end of range, so $s$ is relatively well preserved with depth and the LET distribution remains narrow. 
Further research is required to investigate these important findings in greater detail, at intervening depths and with a larger range of cell types etc.

\section{Treatment Volumes and Normal Tissue sparing.}

The ICRU definition of treatment volumes (GTV, CTV and PTV) are also used for proton therapy. Since the GTV and PTV consists of normal tissue (with some microscopic tumour cells), great care is required to assess if any excess RBE (and consequently dose) beyond the RBE=1.1 used in current treatment planning software systems will influence tissue viability and functionality. It is only in some organs systems that complete loss of function is accepted, and great care is required in the central nervous system, since the low $\alpha / \beta$ of 2 Gy will drive up RBE at low dose per fraction. Outside the PTV, it is equally important to check if any disadvantage due to RBE values exceeding 1.1 will exert any changes in expected side effects. Where there is uncertainty in RBE assessments, the intended dose sparing ratio between the PTV dose and an organ at risk dose outside the PTV should sometimes be improved to accommodate the RBE uncertainty [34]. A further cause of concern is that with scanned proton beams tissues receiving considerably lower doses outside the PTV may have a higher average LET value than within the PTV. In such cases, modelled RBE values could be used to check if tissue tolerances are breached. Tables of LET and RBE values generated by one model for this purpose are available in [35] as supplementary information and a matrix of ICRU volumes and consequences of reduced or increased RBE values is given elsewhere [10].

\section{Discussion: How can radiobiology improve proton therapy?}

Some groups have proposed LET×Dose as being a good predictive index, which bypasses the need for the RBE concept. However, the product does not have meaningful dimensions and RBE becomes a hidden variable (related to each parameter in a different way). As a result, plots of this product against cell survival do not show significant correlation [35] and obscure the potential underlying biology.

The invariant 1.1 RBE value used currently has been extensively criticised on the basis of $L Q$ model theory, use of inappropriate short-term bio-assays and use of non-megavoltage control irradiation. When will the constant RBE be replaced? Will this be left to individual institutions to decide will international bodies (e.g. ICRU and ICRP) intervene or will it be left to the collective accrual of long term toxicity data?

In the case of Carbon ions, but not as yet with protons, the depth-dose profile of each single SOBP field was designed to compensate for the inevitable increase in LET along its path [36]: deeper regions, where the LET is highest, receive less dose; shallower regions are allowed a 
higher dose because of the lower prevalent LET. These principles are shown in figure 5 . Confirmation of a marked increase in RBE for lung fibrosis in patients treated by single SOBP proton fields has increased this concern (Underwood et al 2017, personal communication). The weighting of proton dose towards the distal end of the beam should be reduced to compensate for such an effect.

A major challenge faces every proton therapy clinician: should they adopt different RBE values to limit toxicity and ensure better effectiveness in some situations, despite the inherent uncertainty in RBE values? Should randomised control studies be done to test RBE allocations? For example, by allowing randomisation of patients to either the standard RBE or to an RBE of 1.2 in the CNS normal tissues [10]. Also, very radiosensitive tumours with very high $\alpha / \beta$ ratios and correspondingly very low RBEs, such as medulloblastoma where $\alpha / \beta=28$ Gy, have an estimated RBE of 1.06 (from the model used to generate figures 3(a-c)). In such situations, a tumour RBE reduction of only $1.03-1.05$, or not using an RBE at all could be used, provided that critical structures are not overdosed, but where an RBE of 1.2 or above might be applied protectively. Such an approach might increase safety and effectiveness.

It remains to be seen if clinicians will adopt these suggestions. Considerable reflection and active involvement in the radiobiological issues will be required by future clinicians who use proton beams.

\section{Future opportunities}

Proton therapy has significant potential in the treatment of cancer, but must be used carefully with respect given to the underpinning physics and radiobiology. It is imperative that further preclinical research and model development aims to predict and quantify the optimal treatment approaches for proton therapy so that their full potential can be realized in the advancing era of personalized medicine.

\section{Acknowledgements}

KMP acknowledges support from MRC (G1100014/1) and EPSRC (Ep/K022415/1). 


\section{Tables and Figures}

Table 1: The linear quadratic model adapted for high LET radiations

The bioeffectiveness $E$ is given by
$E=\alpha d+\beta d^{2}$, where $\alpha$ and $\beta$ are radio-sensitivity coefficients, $\alpha$ predominating at
low dose and $\beta$ at high dose. For the same bio-effect, protons and conventional
megavoltage x-rays (photons) can be represented as:
proton effect $=x$-ray (photon) effect,
or
$E=\alpha_{H} d_{H}+\beta_{H} d_{H}^{2}=\alpha_{L} d_{L}+\beta_{L} d_{L}^{2}$
where subscripts $L$ and $H$ refer to the lowest and higher LET radiations. The RBE
is then the dose ratio $d_{L} / d_{H}$, which should always exceed 1. These relationships can
be seen in figure 1, where it can also be noted that the RBE is larger at lower dose
and effectiveness levels (this is due to the linear quadratic shapes of each curve).
The Biological effective dose (BED) is then
approach.
reference low $L E T d_{H}\left(R B E m a x+\frac{R B E m i n^{2} d_{H}}{\left(\frac{\alpha}{\beta}\right)}\right)_{L}$ ratio (see [35]).




\section{Figures}

Figure 1: Demonstration of the RBE principle for two dose effectiveness curves produced by X-rays (photons) and protons (assuming $\alpha_{L}=0.15 \mathrm{~Gy}^{-1}, \alpha_{H}=0.24 \mathrm{~Gy}^{-1}, \beta_{\mathrm{L}}=0.03 \mathrm{~Gy}^{-2}, \beta_{\mathrm{H}}=0.032$ Gy-2) and where two different iso-effect levels [1] and [2] are considered and their corresponding RBEs are shown. The numbers 3, 3.8, 5 and 6 refer to the physical doses where each iso-effect line meets each curve and so are respectively $d_{H}$ followed by $d_{L}$ for isoeffect [1] and $d_{H}$ and $d_{L}$ for isoeffect [2]; the RBE is given by $d_{L} / d_{H}$ in each case and is shown on top of the frame. (Reproduced from [37])

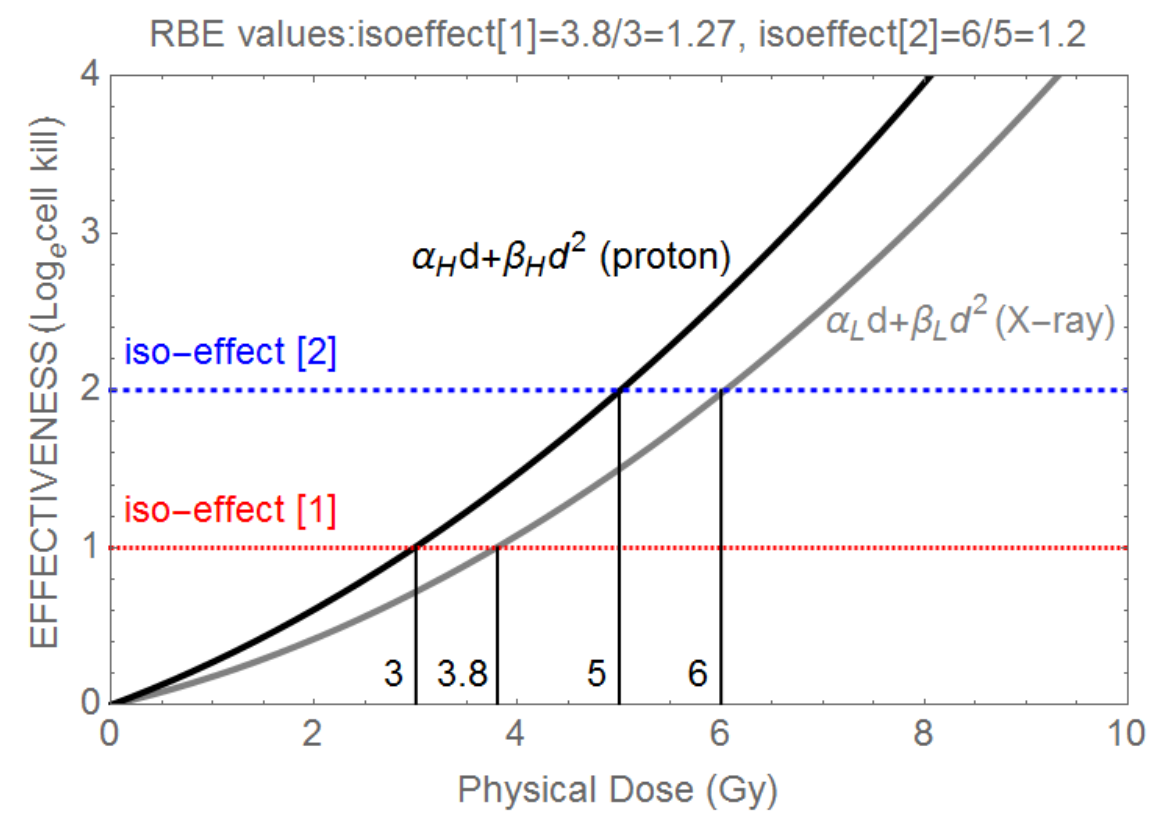


Figure 2 Comparison of relative biological effectiveness (RBE) weighted or biological dose (the product of physical dose and RBE, which is expressed as Gy(RBE), calculated using clinical constant and experimental variable RBE values. Application of variable RBE resulted in an increase of RBE weighted dose in the tumour region (ie, spread-out Bragg peak plateau). Experimental data was obtained from cells irradiated at various positions on the SOBP. A comparison of the Physical and RBE-weighted dose versus depth for a $220 \mathrm{MeV}$ single fraction proton beam. b) Measurement of cell killing RBE relative to $225 \mathrm{kVp}$ X-rays as a function of proton dose per fraction at 4 experimental positions on the SOBP. Analysis of data adapted from [20]
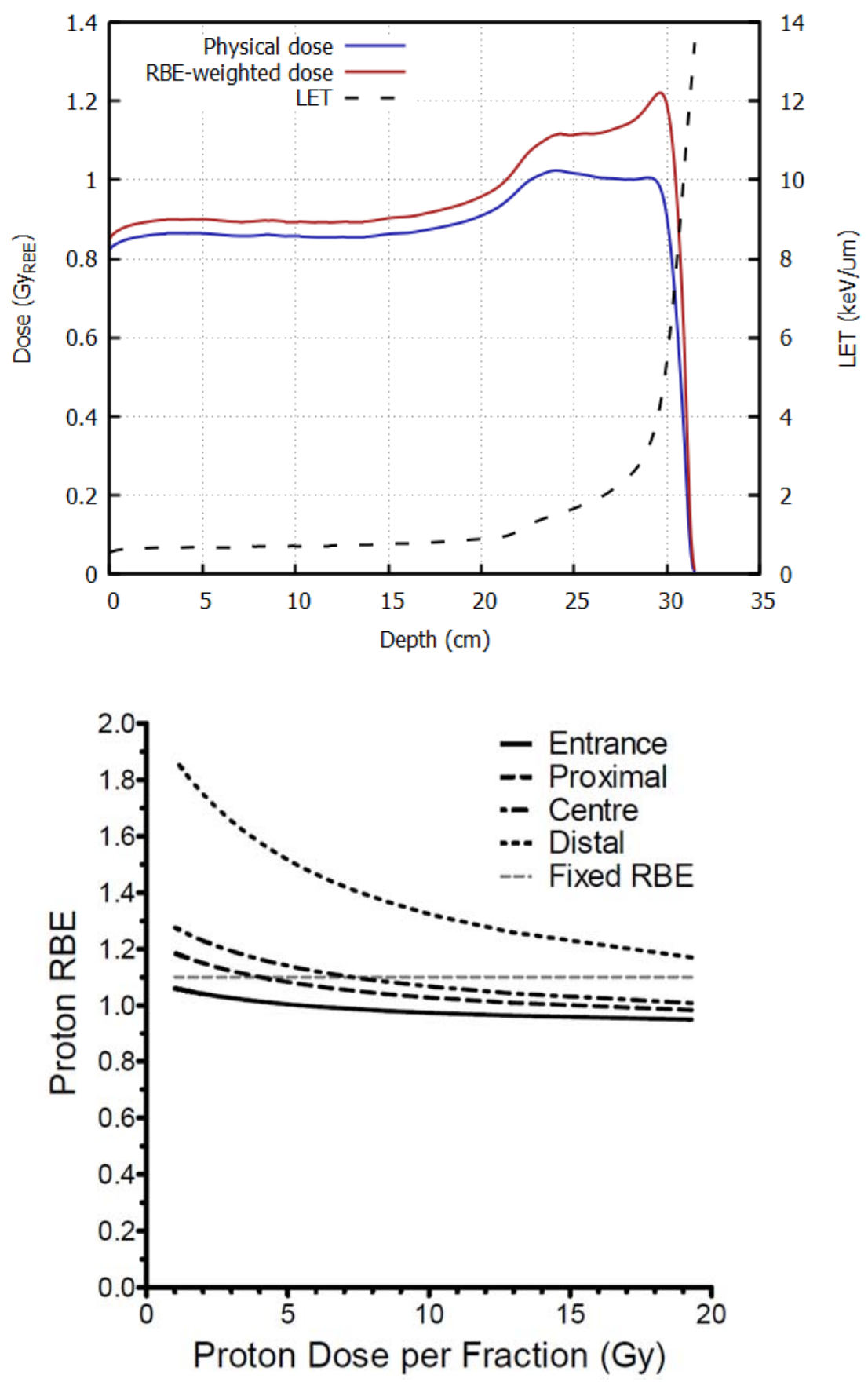
Figure 3 (a-c): three examples of estimated RBE at different proton dose per fraction, for (a) LET of $1.1 \mathrm{keV} . \mu \mathrm{m}^{-1}$, (b) $1.8 \mathrm{keV} \cdot \mu \mathrm{m}^{-1}$. (c) $3.6 \mathrm{keV} \cdot \mu \mathrm{m}^{-1}$. It should be noted that (a) and (b) are typical Let values within spread out Bragg peaks, whereas (c) will be found towards the end of end of Spread out Bragg peaks and even in normal tissue outside the PTV when scanned beams are used.

(a)

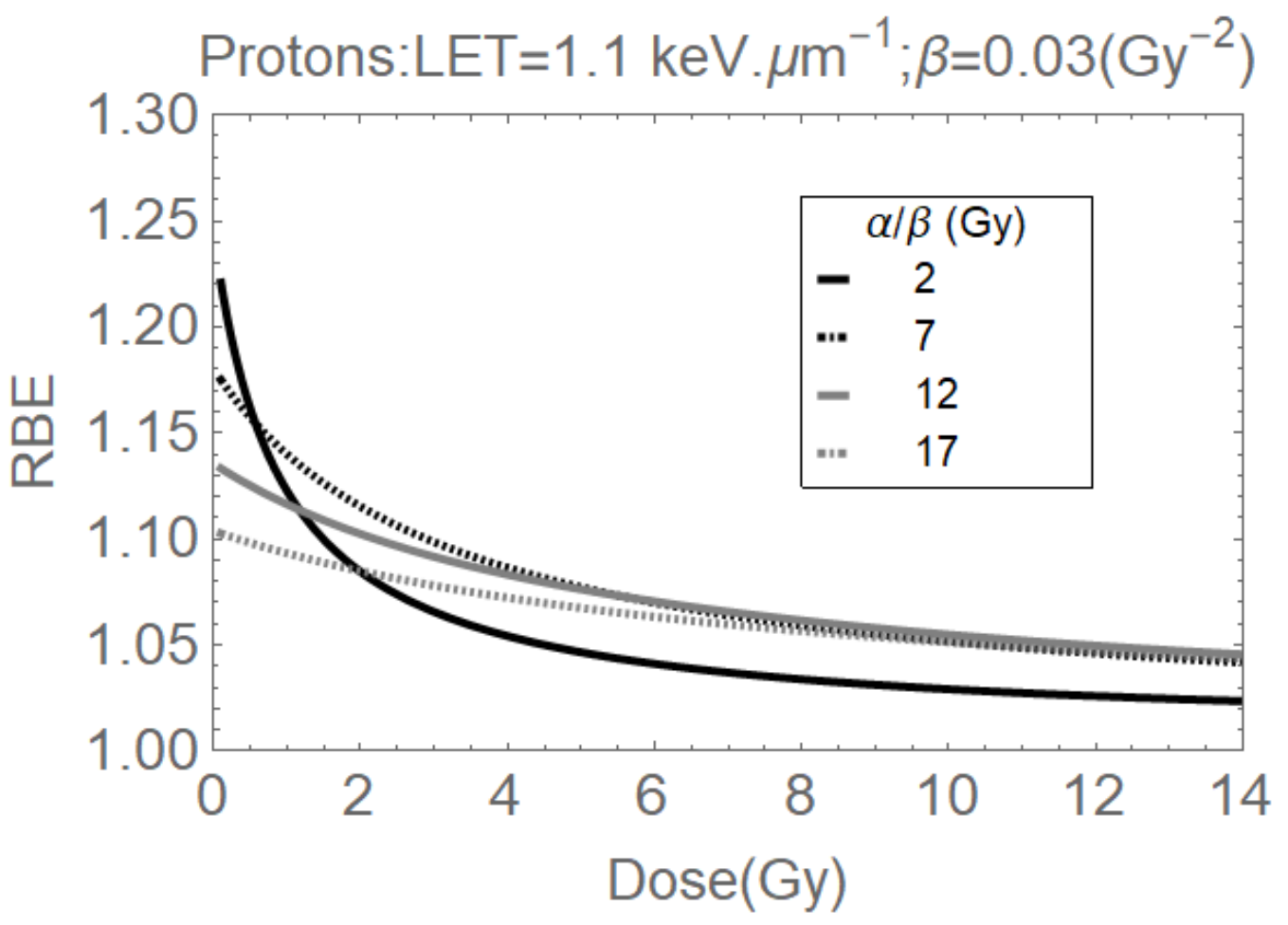


(b)

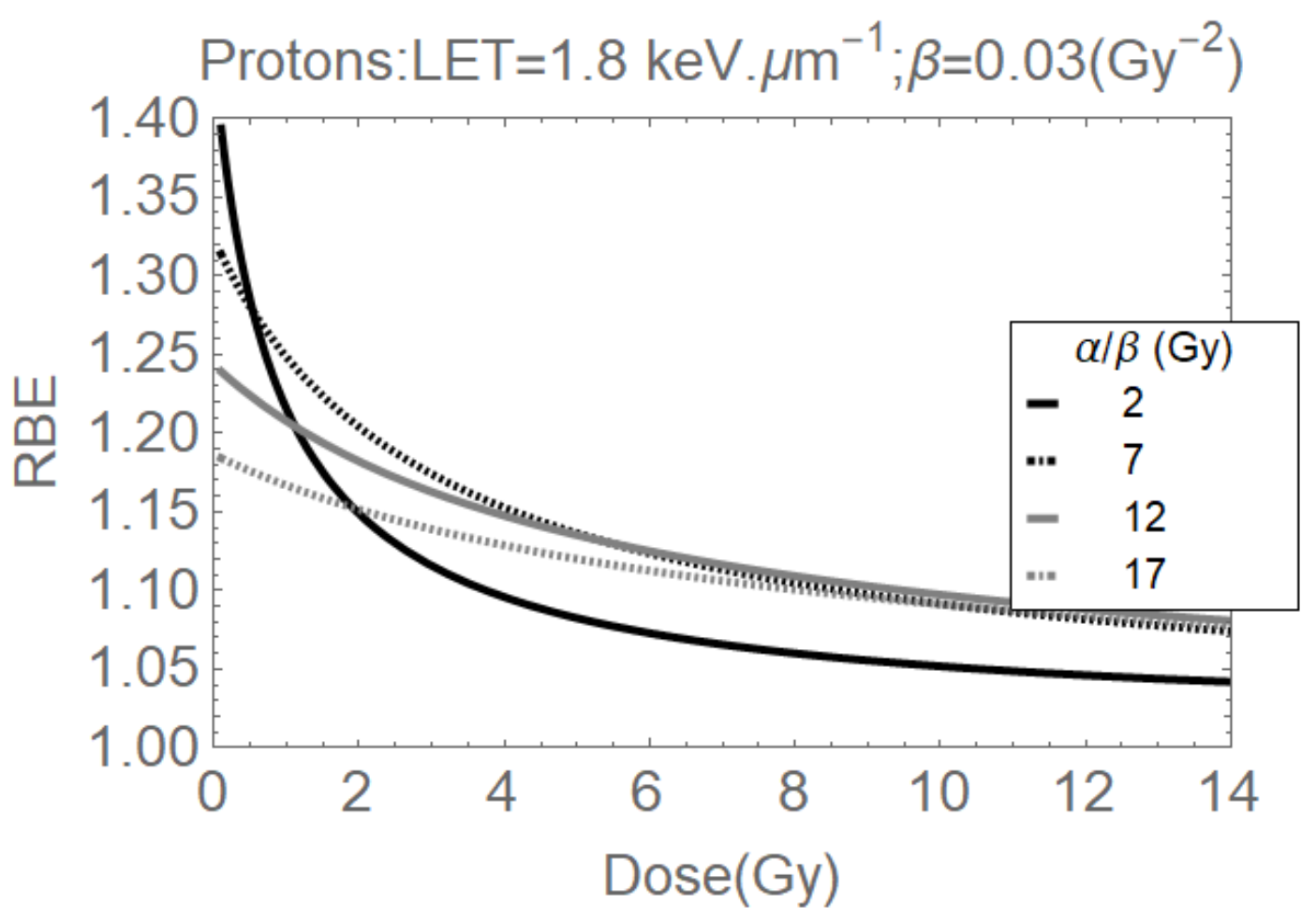

(c)

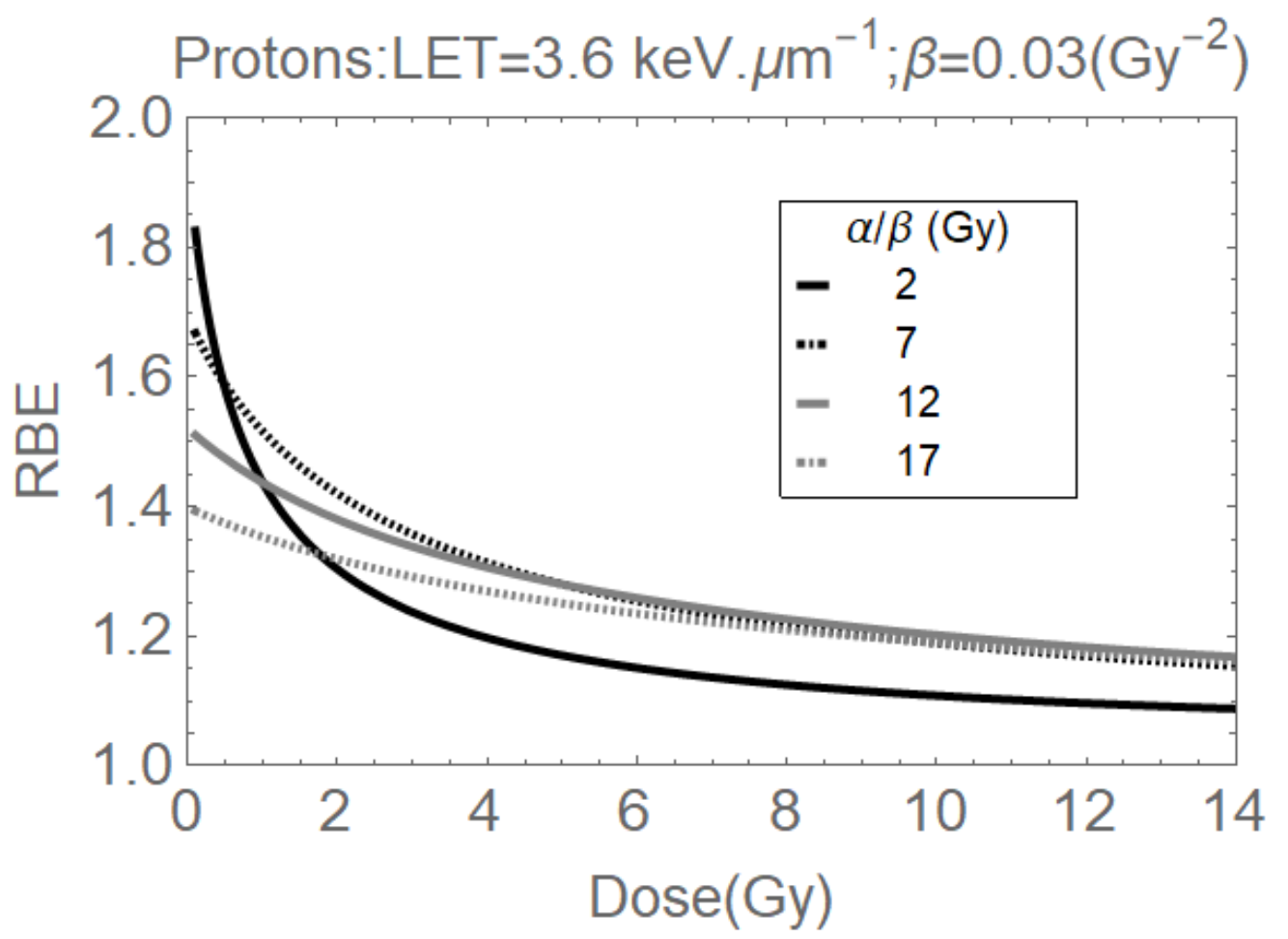


Figure 4. Modelled relationship between RBE with depth at the mid-SOBP and distal SOBP obtained from data averaged for two human cell lines (HeLa and a head and neck squamous cell cancer line) for Caesium and Cobalt referenced RBE values by [33] and assuming RBE falls to around 1.02-1.03 which is consistent with 1 in the biological experiments. (reproduced from [6])

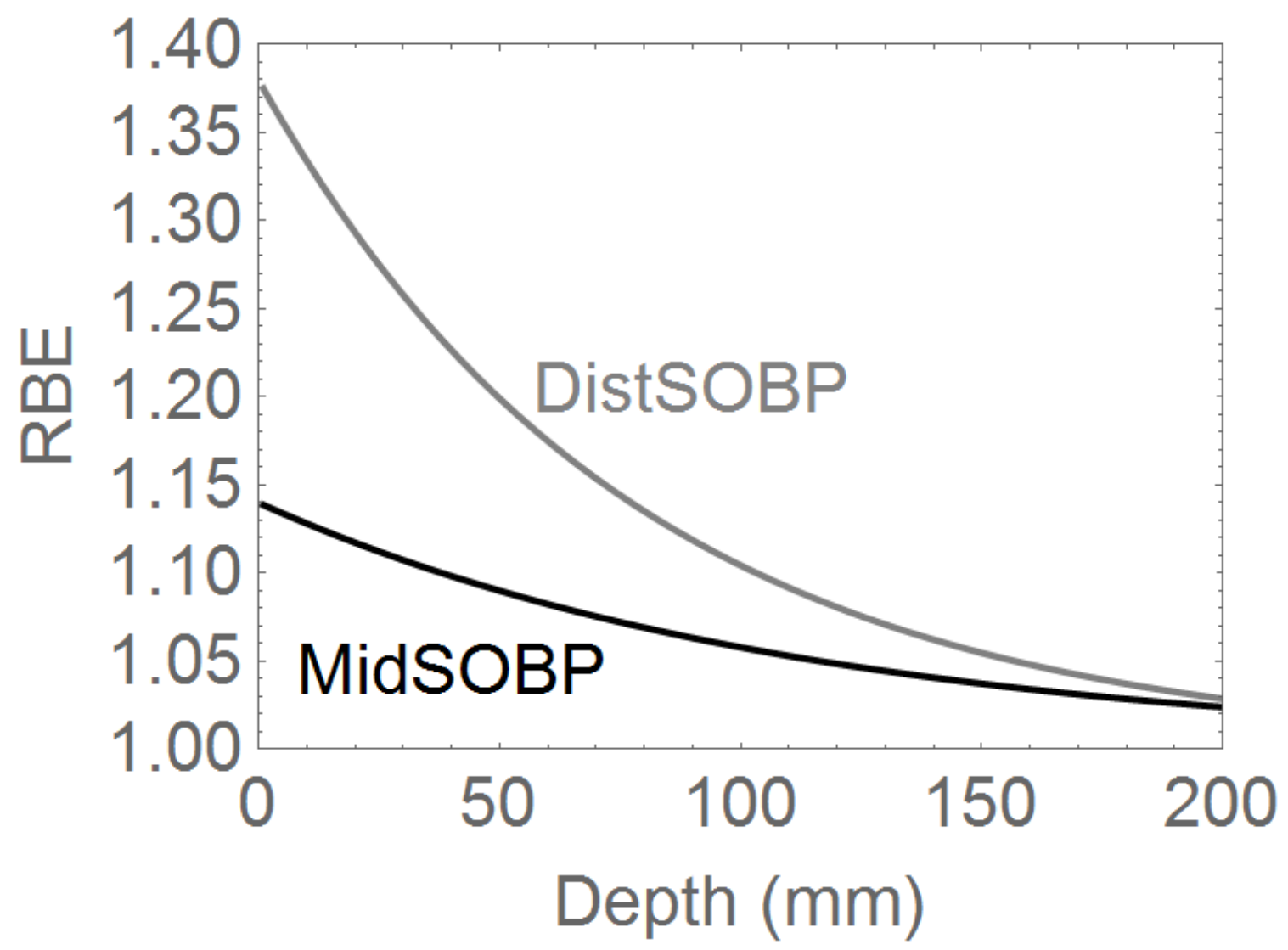


Figure 5: Schematic diagram, based on concept in [36] of how a deliberately designed dose profile (blue) can be used to compensate for increasing LET (black) and RBE (brown) with depth, the net effect being a uniform BED across the target volume (red).

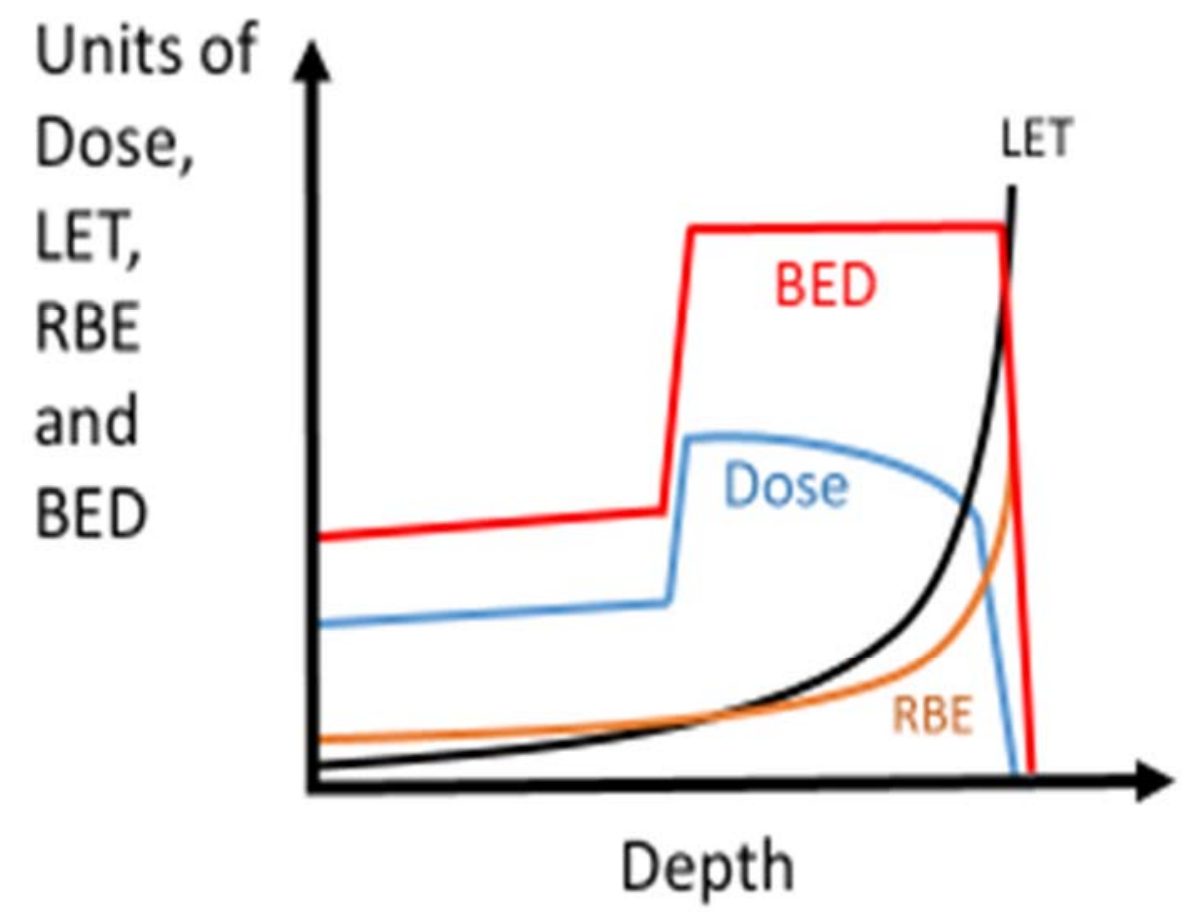




\section{References}

[1] Sharma RA, Plummer R, Stock JK, Greenhalgh TA, Ataman O, Kelly S, et al. Clinical development of new drug-radiotherapy combinations. Nature reviews Clinical oncology. 2016;13:627-42.

[2] Jones B, Dale RG, Deehan C, Hopkins KI, Morgan DA. The role of biologically effective dose (BED) in clinical oncology. Clinical oncology. 2001;13:71-81.

[3] Grassberger C, Trofimov A, Lomax A, Paganetti H. Variations in linear energy transfer within clinical proton therapy fields and the potential for biological treatment planning. International journal of radiation oncology, biology, physics. 2011;80:1559-66.

[4] Barendsen GW. Responses of cultured cells, tumours and normal tissues to radiations of different linear energy transfer. Current Topics in Radiation Research Quarterly. 1968;4:293-356.

[5] Hill MA. The variation in biological effectiveness of $X$-rays and gamma rays with energy. Radiation protection dosimetry. 2004;112:471-81.

[6] Jones B. Historical development of radiotherapy including what was learnt from fast neutrons. Practical Radiobiology for Proton Therapy Treatment Planning. Bristol and Philadelphia: Institute of Physics Publishing; 2017. p. 5-4 - 5-7.

[7] Skarsgard LD. Radiobiology with heavy charged particles: a historical review. Physica medica : PM : an international journal devoted to the applications of physics to medicine and biology : official journal of the Italian Association of Biomedical Physics. 1998;14 Suppl 1:1-19.

[8] Todd P. Heavy-ion irradiation of cultured human cells. Radiation research Supplement. 1967;7:196207.

[9] Weyrather WK, Ritter S, Scholz M, Kraft G. RBE for carbon track-segment irradiation in cell lines of differing repair capacity. International journal of radiation biology. 1999;75:1357-64.

[10] Jones B. Towards Achieving the Full Clinical Potential of Proton Therapy by Inclusion of LET and RBE Models. Cancers. 2015;7:460-80.

[11] Paganetti H. Relative biological effectiveness (RBE) values for proton beam therapy. Variations as a function of biological endpoint, dose, and linear energy transfer. Physics in medicine and biology. 2014;59:R419-72.

[12] Paganetti H, Niemierko A, Ancukiewicz M, Gerweck LE, Goitein M, Loeffler JS, et al. Relative biological effectiveness (RBE) values for proton beam therapy. International journal of radiation oncology, biology, physics. 2002;53:407-21.

[13] Chaudhary P, Marshall TI, Currell FJ, Kacperek A, Schettino G, Prise KM. Variations in the Processing of DNA Double-Strand Breaks Along 60-MeV Therapeutic Proton Beams. International journal of radiation oncology, biology, physics. 2016;95:86-94.

[14] Chaudhary P, Marshall TI, Perozziello FM, Manti L, Currell FJ, Hanton F, et al. Relative biological effectiveness variation along monoenergetic and modulated Bragg peaks of a $62-\mathrm{MeV}$ therapeutic proton beam: a preclinical assessment. International journal of radiation oncology, biology, physics. 2014;90:27-35.

[15] Dasu A, Toma-Dasu I. Impact of variable RBE on proton fractionation. Medical physics. 2013;40:011705.

[16] Jones B. Why RBE must be a variable and not a constant in proton therapy. The British journal of radiology. 2016;89:20160116.

[17] Mohan R, Peeler CR, Guan F, Bronk L, Cao W, Grosshans DR. Radiobiological issues in proton therapy. Acta oncologica. 2017;56:1367-73.

[18] Tommasino F, Durante M. Proton radiobiology. Cancers. 2015;7:353-81.

[19] Britten RA, Nazaryan V, Davis LK, Klein SB, Nichiporov D, Mendonca MS, et al. Variations in the RBE for cell killing along the depth-dose profile of a modulated proton therapy beam. Radiation research. 2013;179:21-8.

[20] Marshall TI, Chaudhary P, Michaelidesova A, Vachelova J, Davidkova M, Vondracek V, et al. Investigating the Implications of a Variable RBE on Proton Dose Fractionation Across a Clinical Pencil 
Beam Scanned Spread-Out Bragg Peak. International journal of radiation oncology, biology, physics. 2016;95:70-7.

[21] Liu Q, Ghosh P, Magpayo N, Testa M, Tang S, Gheorghiu L, et al. Lung cancer cell line screen links fanconi anemia/BRCA pathway defects to increased relative biological effectiveness of proton radiation. International journal of radiation oncology, biology, physics. 2015;91:1081-9.

[22] Fontana AO, Augsburger MA, Grosse N, Guckenberger M, Lomax AJ, Sartori AA, et al. Differential DNA repair pathway choice in cancer cells after proton- and photon-irradiation. Radiotherapy and oncology : journal of the European Society for Therapeutic Radiology and Oncology. 2015;116:374-80. [23] Nickoloff JA, Jones D, Lee SH, Williamson EA, Hromas R. Drugging the Cancers Addicted to DNA Repair. Journal of the National Cancer Institute. 2017;109.

[24] Carabe-Fernandez A, Dale RG, Jones B. The incorporation of the concept of minimum RBE (RbEmin) into the linear-quadratic model and the potential for improved radiobiological analysis of high-LET treatments. International journal of radiation biology. 2007;83:27-39.

[25] McNamara AL, Schuemann J, Paganetti H. A phenomenological relative biological effectiveness (RBE) model for proton therapy based on all published in vitro cell survival data. Physics in medicine and biology. 2015;60:8399-416.

[26] Wedenberg M, Lind BK, Hardemark B. A model for the relative biological effectiveness of protons: the tissue specific parameter alpha/beta of photons is a predictor for the sensitivity to LET changes. Acta oncologica. 2013;52:580-8.

[27] Gunther JR, Sato M, Chintagumpala M, Ketonen L, Jones JY, Allen PK, et al. Imaging Changes in Pediatric Intracranial Ependymoma Patients Treated With Proton Beam Radiation Therapy Compared to Intensity Modulated Radiation Therapy. International journal of radiation oncology, biology, physics. 2015;93:54-63.

[28] Indelicato DJ, Flampouri S, Rotondo RL, Bradley JA, Morris CG, Aldana PR, et al. Incidence and dosimetric parameters of pediatric brainstem toxicity following proton therapy. Acta oncologica. 2014;53:1298-304.

[29] Peeler CR, Mirkovic D, Titt U, Blanchard P, Gunther JR, Mahajan A, et al. Clinical evidence of variable proton biological effectiveness in pediatric patients treated for ependymoma. Radiotherapy and Oncology. 2016;121:395-401.

[30] Weber DC, Malyapa R, Albertini F, Bolsi A, Kliebsch U, Walser M, et al. Long term outcomes of patients with skull-base low-grade chondrosarcoma and chordoma patients treated with pencil beam scanning proton therapy. Radiotherapy and oncology : journal of the European Society for Therapeutic Radiology and Oncology. 2016;120:169-74.

[31] Mizumoto M, Murayama S, Akimoto T, Demizu Y, Fukushima T, Ishida Y, et al. Long-term followup after proton beam therapy for pediatric tumors: a Japanese national survey. Cancer science. 2017;108:444-7.

[32] Mohan R, Das IJ, Ling CC. Empowering Intensity Modulated Proton Therapy Through Physics and Technology: An Overview. International journal of radiation oncology, biology, physics. 2017;99:30416.

[33] Calugaru V, Nauraye C, Noel G, Giocanti N, Favaudon V, Megnin-Chanet F. Radiobiological characterization of two therapeutic proton beams with different initial energy spectra used at the Institut Curie Proton Therapy Center in Orsay. International journal of radiation oncology, biology, physics. 2011;81:1136-43.

[34] Jones B, Underwood TS, Dale RG. The potential impact of relative biological effectiveness uncertainty on charged particle treatment prescriptions. The British journal of radiology. 2011;84 Spec No 1:S61-9.

[35] Jones B. Clinical radiobiology of proton therapy: modeling of RBE. Acta oncologica. 2017;56:13748.

[36] Kanai T, Furusawa Y, Fukutsu K, Itsukaichi H, Eguchi-Kasai K, Ohara H. Irradiation of mixed beam and design of spread-out Bragg peak for heavy-ion radiotherapy. Radiation research. 1997;147:78-85. [37] Jones B. Proton radiobiology and its clinical implications. Ecancermedicalscience. 2017;11:777. 
